\title{
La question du « déjà écrit » dans le processus d'écriture observé en temps réel
}

Une contribution de la génétique à la didactique

\section{Christophe Leblay}

\section{(2) OpenEdition}

\section{Journals}

Édition électronique

URL : https://journals.openedition.org/ml/343

DOI : $10.4000 / \mathrm{ml} .343$

ISSN : 2274-0511

\section{Éditeur}

Association Modèles linguistiques

Édition imprimée

Date de publication : 1 janvier 2009

Pagination : 153-176

\section{Référence électronique}

Christophe Leblay, «La question du « déjà écrit » dans le processus d'écriture observé en temps réel », Modèles linguistiques [En ligne], 59 | 2009, mis en ligne le 29 août 2013, consulté le 01 juillet 2021.

URL : http://journals.openedition.org/ml/343 ; DOI : https://doi.org/10.4000/ml.343 


\section{La question du " déjà écrit » dans le processus d'écriture observé en temps réel Une contribution de la génétique à la didactique \\ Christophe Leblay}

Devant les exigences persistantes d'un enseignement de l'écriture, et non plus seulement d'un enseignement de l'écrit, les différentes approches disciplinaires dialoguent de plus en plus souvent au point de parvenir à mettre en place, non seulement des lieux ${ }^{1}$ de rencontres réguliers, mais aussi des outils innovants.

Ainsi, à partir des travaux réalisés dans le cadre de l'ITEM $^{2}$, la génétique de l'écriture a permis la promotion de la notion d'avant-texte en production verbale écrite. Cet avant-texte associé à des logiciels ${ }^{3}$ d'enregistrement, développés dans le cadre de la psychologie cognitive, autorise enfin une mise en relief spectaculaire des opérations d'écriture saisies en temps réel.

Il convient d'insister sur l'idée essentielle de la complémentarité des disciplines en question, sans chercher à privilégier une discipline au détriment d'une autre. Il s'agit alors de transposer, et non pas seulement d'appliquer, une méthodologie à une didactique des langues. Ainsi, plus particulièrement, il s'agit de transposer la méthodologie de la critique génétique, via des logiciels de temps réel, à la didactique de la production verbale écrite et plus précisément à la didactique de la production verbale écrite en langues (maternelle, étrangère et seconde).

A partir de modèles de transcription, pouvant rendre compte du rôle essentiel joué par les opérations d'écriture au sein du processus d'écriture, le rôle spécifique du déjà écrit se présente comme un facteur déterminant dans une didactique qui serait vouée à l'écriture : il autorise de mettre en

1. Le Groupe de Recherche sur la Production Verbale Ecrite (GDR PVE - CNRS 2657) est un parfait exemple de ce genre de rencontres pluridisciplinaires où didacticiens, généticiens, linguistes et psycholinguistes se mettent autour $\mathrm{d}^{\prime}$ une table commune. Avec, en prime, des propositions de nouveaux outils pour la didactique de la production écrite.

2. L'ITEM est l'Institut des Textes et Manuscrits Modernes (unité mixte ENSCNRS) : deux équipes transversales, (Equipes «Manuscrits, Linguistique et Cognition» et "Génétique et théories linguistiques») ont cette volonté de diffusion des acquis de la génétique.

3. Voir note 8, p. 156. 
parallèle les opérations qui s'inscrivent à la suite du déjà écrit et les opérations d'écriture qui font retour dans le déjà écrit. Autrement dit, le déjà dit permet une appréhension et une compréhension du phénomène de révision.

\section{Les opérations d'écriture et le mouvement du curseur saisis en temps réel}

Avant de parvenir à tenter une description des opérations d'écriture associées aux mouvements du curseur, laquelle forme un échantillon exemplaire $\mathrm{d}$ 'un avant-texte saisi en temps réel, il semblerait toutefois judicieux de faire retour sur les gestes d'écriture.

\subsection{Les gestes d'écrire}

Le geste scriptural est un geste variable selon les époques : il semble, par nature, induit par l'outil, par l'objet technique (Velay 2004, 37) dont le degré zéro pourrait être représenté par un doigt qui fait des traces sur le sable. A ceux qui se plaisent à opposer ce qui serait un mode d'écrire non technologique, représenté par l'utilisation du support du papier et du crayon/stylo, à ce qui serait un mode technologique, représenté par l'utilisation du support de l'écran/clavier, il ne faut cesser de rappeler que le papier est un support de haute technologie au même titre que le support de l'écran. Choisir donc d'écrire, via un clavier, via un écran, ne veut pas dire choisir une technologie par rapport à ce que serait une non technologie représentée par le papier. Il s'agit de fait de choisir entre une technologie et une autre technologie, de faire le choix entre la technologie du papier, du stylo et la technologie de l'écran, du clavier (et des tablettes graphiques), et bientôt de l'écran tactile avec son clavier incorporé.

Mais si la question du choix du support technique se pose pour une génération de personnes qui ont pris l'habitude effective et affective d'écrire (de lire et de penser) à partir du support qu'est le papier (il leur est expressément demandé de changer d'outils), elle ne se pose pas pour les plus jeunes, pour lesquels il n'y a pas de transfert technologique : le choix de l'outil ne se pose même pas, tant il s'est imposé par lui-même. Ecrire (et lire) reste un geste qui se fait naturellement avec un écran ${ }^{4}$ et son clavier.

Les différentes manières d'appréhender corporellement ce geste d'écrire se résument schématiquement à trois (tableau1) :

4. Il est bien tentant de faire un parallèle entre deux machines : la machine à compter et la machine à écrire. La première a depuis déjà un certain temps intégré nos manières d'agir ; qui viendrait, par exemple, contester la présence des calculatrices lors de certaines épreuves scientifiques du Baccalauréat? La seconde a encore devant elle une longue marche; à quand un Baccalauréat de langues rédigé sur traitement de texte? 


\begin{tabular}{|c|c|c|c|}
\hline \multirow{5}{*}{ 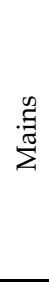 } & \multirow{2}{*}{ Manipulation du clavier } & \multicolumn{2}{|c|}{ Opérations d'écriture } \\
\hline & & \multicolumn{2}{|c|}{ Pause dans l'écriture } \\
\hline & \multirow{3}{*}{$\begin{array}{c}\text { Manipulation } \\
\text { de périphériques d'entrée }\end{array}$} & Souris & \multirow{3}{*}{$\begin{array}{c}\text { Déplacements } \\
\text { du curseur }\end{array}$} \\
\hline & & Flèches & \\
\hline & & Pavé tactile & \\
\hline \multirow{2}{*}{$\begin{array}{l}\stackrel{㐅}{ٍ ~} \\
ٍ\end{array}$} & \multirow{2}{*}{ Mouvement oculaire } & \multicolumn{2}{|c|}{ Ecran } \\
\hline & & \multicolumn{2}{|c|}{ Clavier/souris/tablette graphique } \\
\hline $\begin{array}{l}. x \\
0 \\
\end{array}$ & Emission & & \\
\hline
\end{tabular}

Tableau 1.

Manières d'appréhension du geste d'écrire

D'une manière générale, ce tableau ne fait que rappeler la grande relativité des propos liés à l'écriture : ce qui est vrai aujourd'hui ne le sera qu'un peu moins demain, tant le développement des outils de demain permettra de préciser ce qui reste encore dans le flou aujourd'hui. Et ce qui est vrai de la difficulté à changer d'outils, de la difficulté à appréhender physiquement un (nouveau) geste d'écriture, l'est tout aussi de la difficulté à analyser des nouvelles pratiques d'écriture.

Des trois lieux d'appréhension corporelle (colonne de gauche) impliqués dans l'activité scripturale, le rôle des mains est, pour l'heure actuelle, central : il est possible d'avoir une activité scripturale sans utiliser ses yeux. Les pratiques des scripteurs mal voyants et non voyants, usant d'outils spécifiques mis à leurs dispositions, ne font que le confirmer ${ }^{5}$. Quant à la voix, elle est en train de faire une apparition récente dans le domaine de la production écrite.

Il est possible de dire, en se référant à la deuxième colonne, que le développement technique oriente l'écriture vers une activité de plus en plus subtile ${ }^{6}$ au point où l'émission de la voix permet déjà, dans certains contextes, de se passer complètement de la manipulation.

5. Les informations données par le suivi des yeux lors de productions écrites restent, pour l'instant, sans équivalent. Ainsi mettre en évidence la fonction des pauses dans l'écriture sans pouvoir suivre le déplacement des yeux reste une démarche vaine tant la corrélation entre les yeux et les pauses semble faire sens.

6. Pour s'en convaincre, il suffit de prendre en considération les verbes employés, dans les manuels d'utilisateur pour traitements de texte et messageries pour mobiles, pour suggérer le geste d'écriture : ainsi de «frapper " pour décrire ce qui se passe au clavier d'un ordinateur, on est en train de passer à «tapoter», 
Ecrire sera possible à partir de la voix, car écrire reste fondamentalement un dire (Leblay 2007a, 102). Paradoxalement, il est ainsi aisé de voir comment, malgré le développement technique des outils d'écriture vers ce plus subtile, la présence et le rôle de la voix du scripteur, alors énonciateur, reste prédéterminante dans le geste d'écrire, que cette voix soit intériorisée, comme dans le cas d'une écriture produite avec le recours des yeux et/ou des mains, ou bien enfin, totalement extériorisée, à partir des techniques de reconnaissance vocale.

La présente description se fait à partir de la manipulation du clavier et de certains périphériques sans prendre en compte du travail bien réel des mouvements oculaires nécessaires pour pouvoir effectuer ces manipulations. Le rôle des yeux est effectif sans être, pour autant, pris en compte.

\subsection{L'avant-texte saisi en temps réel}

Depuis le travail fondateur ${ }^{7}$ de Claudine Fabre (1987), consacré à l'étude génétique des avant-textes scolaires, et, à partir des travaux de Claire Doquet-Lacoste (2004) relatif au temps réel des mêmes avant-textes, il est maintenant bien admis que, à l'image de ce qui s'est passé dans les approches de l'oral où l'enregistrement des productions était devenu indispensable, enregistrer la production écrite est essentiel pour (mieux) l'étudier. Les logiciels dédiés à l'enregistrement de la production écrite sont le plus souvent même directement accessibles depuis l'Internet ${ }^{8}$.

$\mathrm{Au}$ sein des pratiques du dire', l'avant-texte reste une activité qui permet de passer du langage en acte à l'écriture en acte. Il désigne, selon les mots de Chanquoy et Fenoglio $(2007,5)$ «tout ce qui se passe avant la production du texte écrit et dont les traces attestent d'une mise en acte cognitive et graphique directement liée au texte final produit. »

Cet avant-texte, sous sa forme d'écriture en acte, peut être saisi en temps réel : il est alors décrit à partir des opérations d'écriture, des pauses dans l'écriture, du suivi oculaire et des mouvements du curseur (Tableau 2).

«toucher » et « effleurer» pour rendre compte du geste de la main, ou des doigts, ou même d'un seul doigt, sur le clavier tactile des téléphones mobiles.

7. Travail auquel il faut associer celui de Catherine Boré (2000).

8. Pour se limiter aux plus connus : Eye and Pen (www.eyeandpen.net.), Inputlog (www.inputlog.net), JEdit (www.nada.kth.se/iplab/trace-il/index.html), ScriptLogPro (www.scriptlog.net) et Translog (www.translog.dk).

9. Dire de préférence à faire : faire suggère acte de langage, tandis que dire suggère langage en acte. (Leblay 2007a, 102) 


\begin{tabular}{|c|c|c|c|c|c|}
\hline \multicolumn{7}{|c|}{ Avant-texte saisi en temps réel } \\
\hline \multirow{2}{|c|}{ Opérations d'écriture } & \multicolumn{2}{|c|}{$\begin{array}{c}\text { Mouvements } \\
\text { du curseur }\end{array}$} & $\begin{array}{c}\text { Pauses } \\
\text { dans l'écriture }\end{array}$ & $\begin{array}{c}\text { Mouvements } \\
\text { des yeux }\end{array}$ \\
\cline { 1 - 1 } Ajout Suppression & \multirow{2}{*}{$\begin{array}{c}\text { Remplacement } \\
\text { \& déplacement }\end{array}$} & $\square$ & $\begin{array}{l}\leftarrow \rightarrow \\
\uparrow \downarrow\end{array}$ & - & - \\
\hline
\end{tabular}

Tableau 2.

L'avant-texte saisi en temps réel

Les mouvements du curseur se manifestent essentiellement par la manipulation de la souris $\mathcal{\beta}$, des flèches $\leftarrow$, ou encore du pavé tactile $\square$. Le choix de l'un ou l'autre périphérique par le scripteur est loin d'être vain et autorise de nombreuses interprétations. Nous verrons, par la suite, l'importance de la distribution de certains gestes au détriment d'autres.

Concernant les pauses dans l'écriture, il serait tentant de les assimiler aux opérations d'écriture tant elles en font partie : elles sont, de fait, le plus souvent, une pause des opérations d'écriture et sont fortement associées au suivi oculaire.

L'approche développée dans ce travail ${ }^{10}$ se concentre sur l'accomplissement des opérations d'écriture par les mouvements du curseur.

\subsection{Les opérations d'écriture et les mouvements du curseur}

Les quatre opérations d'écriture sont, selon Grésillon (1994, 150), au nombre de quatre. Ces opérations sont soit élémentaires (ajout et suppression), soit composées (remplacement et déplacement). Elles sont dites élémentaires parce qu'elles peuvent soit fonctionner en tant que telles, soit entrer en composition et former alors les opérations dites composées $^{11}$.

\section{Les opérations élémentaires : ajout \& suppression}

Il est habituel de commencer par mettre en début de description l'opération dite d'ajout, elle-même représentée par la suite de deux symbôles : $\varnothing \Rightarrow A$. Ce qui revient à dire que l'écriture, initiée par un ajout, commencerait par ce premier symbôle $\varnothing$. Comment alors est-il possible d'interprêter cette représentation ? Que représente ce signe qui note un

10. Le parti pris de ce travail est de chercher à proposer une transposition didactique autant que des outils accessibles. Les apports du suivi oculaire (en relation aux pauses dans l'écriture) sont indéniables mais sont, à l'heure actuelle, attachés à des outils réservés principalement à la recherche. Ils sont encore trop peu accessibles.

11. Le geste de corriger recouvre n'importe quelle opération : on peut corriger en ajoutant, en supprimant, en remplaçant et en déplaçant. 
vide ? Celui-ci représente-t-il ce qui se passe durant la pause originelle de toute opération d'écriture ?

Puis, suit l'opération de suppression, décrite avec les mêmes symboles que ceux utilisés pour l'ajout, mais dans un ordre inversé $(A \Rightarrow \varnothing)$; ce qui pourrait laisser croire que ces deux opérations (ajout et suppression) fonctionnent comme deux opérations placées en vis-à-vis, opposées, et donner une image de l'écriture fonctionnant sur le registre de ce qui est, principalement, ajouté ou supprimé. Ecrire serait alors une succession d'ajouts et de suppressions. Nous verrons par la suite qu'il n'en est rien et que cette représentation symbolique induit le plus souvent en erreur.

\section{Les opérations composées : le cas du remplacement}

Pour l'opération de remplacement, il est commun de la représenter par la formule suivante : $A \Rightarrow B$, où un $A$ devient un $B$. Là encore, il faut se méfier des apparences qui laisseraient croire à une disparition complète d'un $A$ au profit d'un $B$ sans laisser de trace. Une telle description donnerait une idée bien incomplète de la description du fonctionnement du remplacement.

La sémantique du verbe 'remplacer' peut s'entendre de deux manières : soit le sujet désigne l'agent, soit le sujet désigne ce qui prend la place. Dans le premier cas, il marque, de manière non exclusive certes, le fait de créer quelque chose à la place de quelque chose, qui disparaît ainsi complètement; définition qui ressemble beaucoup à la représentation symbolique décrite précédemment où un $A$ disparaît au profit d'un $B$. Dans la seconde acceptation toutefois, le sujet désigne ce qui prend la place et se présente comme synonyme de constructions verbales de type 'se substituer ${ }^{12}$ à' ; ce sujet est alors, d'une manière peu équivoque, noté en référence au temps et prend alors le sens de verbes tels ' (se) succéder à'.

Voilà bien une acceptation qui convient parfaitement au travail visualisé par le temps réel, où il s'agit de noter une succession, une transformation opérée dans le temps entre deux éléments bien plus que de noter une disparition pure et simple d'un élément au profit d'un autre.

\section{La question de la chronologie}

Les études relatives à l'écriture ont pris l'habitude récente de travailler à partir de trois avant-textes bien distincts. C'est précisément à partir de cette triple dimension spatiale de l'écriture qu'il est désormais possible de s'interroger sur la relation parfois mal perçue de l'écriture à sa chronologie.

12. Le terme de substitution est souvent donné pour synonyme à celui de remplacement. Pour plus de détails Leblay $(2007 \mathrm{~b}, 142)$. 


\subsection{La triple dimension (spatiale) de l'écriture}

Aujourd'hui, il existe bien trois types distincts d'avant-texte (tableau 3) : tout d'abord, celui représenté par l'ensemble des manuscrits et tapuscrits, puis celui représenté par les productions numérisées à partir de logiciels de traitement de texte et, enfin, celui des productions enregistrées (donc numérisées) à partir de logiciels de temps réel.

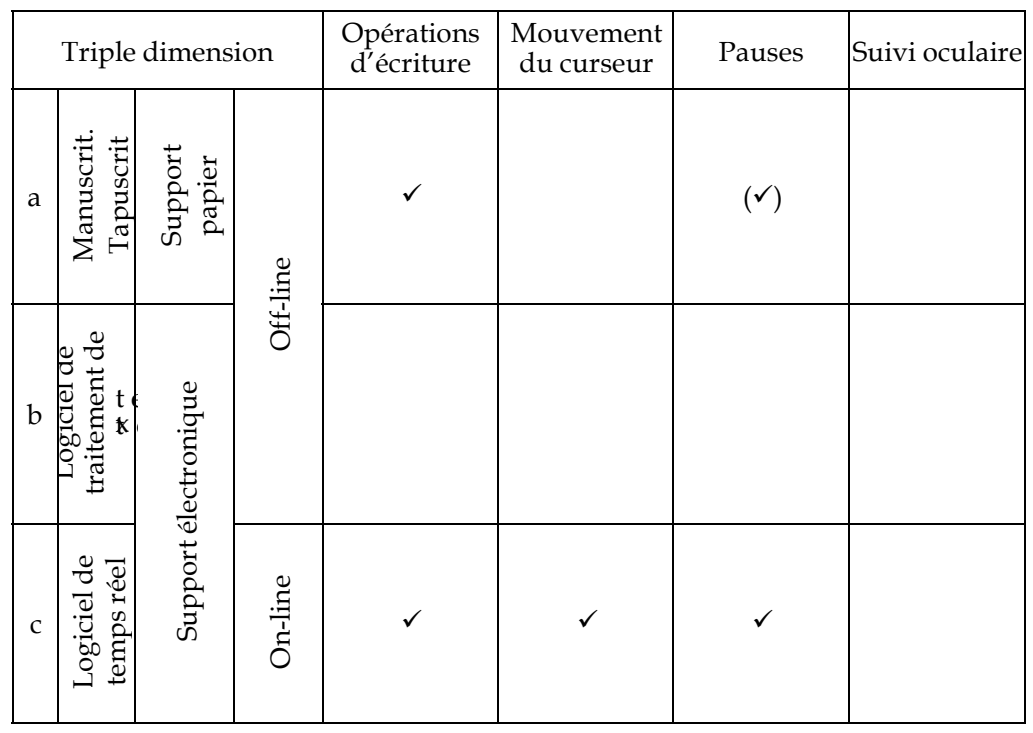

Tableau 3.

Triple dimension spatiale

Sont mis en contraste les trois supports depuis la perspective du support électronique en temps réel puisque c'est bien cette dernière qui permet une description plus exhaustive de l'écriture contemporaine : il est aisé de se rendre compte que, du point de vue des opérations d'écriture, le support papier et le support électronique saisi en temps réel, permettent une saisie réelle des opérations d'écriture, à la différence du support électronique qui annule complètement cette saisie. Dans les études sur papier, sont systématiquement notées les opérations d'écriture ainsi que, $\mathrm{d}^{\prime}$ une certaine manière, les pauses ${ }^{13}$.

Il est devenu tout aussi habituel de se servir de l'expression consacrée $^{14}$ on-line pour opposer ce qui relève uniquement du temps réel aux autres supports désignés alors par l'expression de off-line, que ceux-ci

13. Par exemple, les changements d'encre. Autant d'éléments qui permettent d'induire que le scripteur a fait des pauses dans son écriture.

14. Le choix des expressions on-line et off-line est bien loin d'être satisfaisant en français. Il existe une expression "en ligne" qui pourrait très bien remplacé celle de on-line, mais, pour off-line, « hors ligne » semblerait maladroit. 
soient produits sur papier ou sur support électronique. Il est alors remarquable que l'écriture sur traitement de texte se présente comme un simple support intermédaire ${ }^{15}$, qui, bien que se présentant comme un support électronique (axe $\mathrm{b}-\mathrm{c})$, reste un support off-line, au même titre que le support papier (axe a-b).

Dans l'écriture on-line, sont systématiquement notés, par le logiciel, les opérations d'écriture, les pauses dans l'écriture, les mouvements du curseur et le suivi oculaire, seulement dans le cas où celui-ci est autorisé par le logiciel. Cette écriture on-line ne vient donc pas contredire les études de productions off-line, bien au contraire : les opérations d'écriture étant, dans les deux cas, prises en compte.

Cette question de la dimension spatiale de l'écriture pose alors la question essentielle de la chronologie de l'écriture.

\section{2. Temporalités relative et absolue}

La particularité de ces études on-line tient au fait que l'avant-texte est, à la différence des saisies off-line, systématiquement confronté à sa chronologie. Contrairement aux manuscrits sur papier où la reconstitution semble avant tout être appréhendée à partir du plan visuel, les opérations d'écriture, dans un corpus en temps réel, sont systématiquement appréhendées à partir de leur dimension chronologique, où rien n'est enregistré hors du temps. Cette focalisation sur le temps redonne à l'écriture ce qui avait disparu sans laisser de traces, ou si peu, avec le papier. Elle replace l'écriture dans une dimension temporelle que la technique ne permettait pas de mettre en évidence. Juste retour d'une dimension.

Par principe, toute écriture relève, simultanément, des deux dimensions, et de la dimension spatiale et de la dimension temporelle. Mais, pour des raisons techniques, l'écriture off-line donne priorité à la dimension spatiale, tandis que l'écriture on-line donne priorité à la dimension temporelle. Pour simplifier à l'extrême (tableau 4) :

\begin{tabular}{|c|c|c|c|}
\hline \multicolumn{2}{|c|}{$\begin{array}{c}\text { Dimensions spatiale } \\
\text { vs temporelle }\end{array}$} & Première dimension & $\begin{array}{c}\text { Seconde } \\
\text { dimension }\end{array}$ \\
\hline $\begin{array}{c}\text { Ecriture sur supports papier } \\
\text { et électronique }\end{array}$ & Off-line & $\begin{array}{c}\text { Dimension } \\
\text { spatiale }\end{array}$ & $\begin{array}{c}\text { Dimension } \\
\text { temporelle }\end{array}$ \\
\hline $\begin{array}{c}\text { Ecriture sur support } \\
\text { électronique en temps réel }\end{array}$ & On-line & $\begin{array}{c}\text { Dimension } \\
\text { temporelle }\end{array}$ & $\begin{array}{c}\text { Dimension } \\
\text { spatiale }\end{array}$ \\
\hline
\end{tabular}

Tableau 4

Espace vs temps

15. «Le traitement de texte fait perdre au scripteur - et au linguiste généticien qui voudra l'observer - la richesse et la polyvalence de la trace graphique.» (Grésillon et Lebrave, 2008, 49); ou encore : « L'écriture sur traitement de texte est perpétuel mouvement, mais de ce mouvement les traces nous échappent, et les tirages à l'imprimante livrent paradoxalement une série de clichés lisses, fixations d'instants du texte où ce qui prime est le figement. » (Doquet-Lacoste, 2006, 39). 
Cette prise en compte de la temporalité des opérations d'écriture s'effectue en deux gestes: il s'agit de parvenir, à l'aide de logiciels adaptés, à (se) saisir du mouvement de l'écriture au moment même où il s'effectue sans rien perdre de l'évidence de la succession chronologique des opérations d'écriture, des unes à la suite des autres et des unes par rapport aux autres. Dans un second geste, il s'agit de parvenir, à l'aide de transcriptions adaptées, à (se) visualiser ces mouvements, ces successions en privilégiant la notation de ces opérations successives.

Je propose de nommer temporalité relative le fait de vouloir noter, en priorité, cette succession temporelle relativement aux opérations d'écriture. Mais les logiciels d'enregistrement autorisent (voir annexe) une autre notation : celle d'une temporalité absolue en référence au temps initial. Telle opération est alors notée en lien avec le moment où l'écriture a commencé, c'est-à-dire en lien avec la première interaction avec le clavier ou avec un périphérique.

\section{3. La contiguïté temporelle}

Dans le tableau suivant (5), il est question de pouvoir distinguer de fait l'ajout et la suppression en tant que tels, en tant qu'opérations élémentaires, ou bien en tant qu'opérations entrant en composition.

Comme suggéré précédemment, et donc contrairement à ce qu'une formalisation des opérations d'écriture pourrait laisser croire, l'ajout ne semble pas faire système avec la suppression et offrir l'idée que la production verbale écrite serait construite dans la simple succession des deux gestes élémentaires : ajouter et supprimer. Pas seulement du moins.

Le geste d'ajouter rentrerait dans une distribution complémentaire avec le geste de remplacer. En ce sens, le remplacement forme une alternative théorique à l'ajout pressenti comme geste scriptural rudimentaire de toute production écrite.

\begin{tabular}{|c|c|c|}
\hline \multirow{2}{*}{$\begin{array}{l}\text { Opérations élémentaires, } \\
\text { en un seul temps, } \\
\text { effectuées } \\
\text { à l'aide du clavier, } \\
\text { de la souris } \\
\text { ou du pavé tactile }\end{array}$} & $\frac{+}{2}$ & $\begin{array}{l}(+) \text { ajout } \\
\text { de toute lettre ou de tout segment }\end{array}$ \\
\hline & 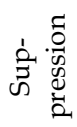 & $\begin{array}{c}(-) \text { suppression } \\
\text { de toute lettre ou de tout segment }\end{array}$ \\
\hline \multirow{2}{*}{$\begin{array}{l}\text { Opérations composées, } \\
\text { en deux temps } \\
\text { effectuées } \\
\text { à l'aide du clavier, } \\
\text { de la souris } \\
\text { ou du pavé tactile }\end{array}$} & 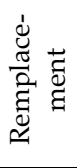 & $\begin{array}{c}\text { (-) suppression } \\
\text { de toute lette ou de tout segment } \\
(+) \text { ajout contigu } \\
\text { d'une autre lettre ou autre segment } \\
\text { au même endroit }\end{array}$ \\
\hline & 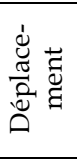 & $\begin{array}{c}\text { (-) suppression } \\
\text { de toute lette ou de tout segment } \\
\sigma(+) \text { ajout contigu } \\
\text { de la même lettre ou même segment } \\
\text { à un autre endroit }\end{array}$ \\
\hline
\end{tabular}

Tableau 5 .

Les opérations d'écriture 
Du point de vue de la temporalité relative qui prend en compte la succession chronologique des opérations d'écriture, il est possible de dire que, d'une manière constitutive, les deux dernières opérations ne peuvent être appréhendées qu'à l'aide des deux premières : pour remplacer et déplacer, il faut inévitablement, et dans un ordre probable mais pas contraint, supprimer et ajouter. Il faut surtout supprimer et, immédiatement après, ajouter : Autrement dit, un ajout, rentrant en composition, doit être contigu à la suppression.

L'idée même de contiguïté fait autant référence au domaine sémantique de l'espace et du temps qu'à celui du relationnel; il s'agit autant de noter une proximité immédiate de deux segments dans la chaîne du discours que de noter une des formes de l'association d'idées, caractérisée par le "rappel successif des états de conscience lorsqu'ils ont été simultanés dans l'espace, ou immédiatement successifs dans le temps » (Legrand ${ }^{16}$, 1972).

\subsection{Temps immédiat et temps différé}

Mais, à regarder de plus près, les choses ne sont pas aussi tranchées qu'elles semblent. Il existe bien un intermédiaire entre la suppression, considéré pour elle-même en tant qu'opération élémentaire, et la suppression entrant en composition.

L'opération de suppression est donc bien une opération plurielle, à trois variables : il est en effet important de faire la différence entre une suppression immédiate, une suppression différée et une suppression entrant en composition pour former, par exemple, un remplacement. J'appelle la première "immédiate » en ce qu'aussitôt apparue, elle est aussitôt supprimée. La seconde n'est qu'un cas particulier de la première: elle est différée en ce sens qu'un autre geste s'est intercalé entre le moment où la suppression immédiate commence à se produire et le moment où cette même suppression immédiate s'achève. Tout n'est pas effacé dans la continuité chronologique d'un même geste, mais en un temps différé parfois très réduit. Ce qui apparaît dans les transcriptions. Ainsi, par exemple, un cas fréquent ${ }^{17}$ emprunté à une production $\mathrm{d}^{\prime}$ une étudiante finnophone:
1) Inscription :
ou vire e $^{<30.40>}$
2) Suppression immédiate de deux élements e' :
ou vire' $<134.08>$
3) Ajout de trois lettres vre:
ou vi/vre ${ }^{<135.13>}$
4) Suppresion en deux temps
ou vi/vre ${ }^{<138.51>/<140.30>}$

- suppression immédiate de vre

- suppression différée des lettres précédentes ou vi

16. Legrand, G. (1972) Dictionnaire de philosophie. Paris, Bruxelles, Montréal: Bordas.

17. Ce qui est apparaît, dans les conventions de transcriptions, avec le caractère barré, sans italique (voir tableau 7). 
En 1) est inscrit ou vire'. En 2) sont immédiatement supprimés deux éléments $e^{\prime}$. En 3) sont ajoutées les lettres vre. A noter que cette suppression successivement suivie d'un ajout forme un remplacement. En 4) sont immédiatement supprimées les lettres vre, de même que, en un temps différé les lettres ou vi. Celles-ci illustrent le cas particulier de la suppression en temps différé : entre le temps où elles ont été inscrites (130.40) et celui où elles ont été supprimées (140.30) s'est écoulé un peu moins de dix secondes.

La concurrence manifeste entre le geste de supprimer et celui de remplacer reste révélatrice des enjeux qui se déroulent sur la page ou sur l'écran. Il reste relativement établi que la suppression pure et dure d'éléments (lettres ou segments) est peu fréquente.

\section{Le déjà écrit}

Sur le modèle constructiviste ${ }^{18}$ selon lequel «appprendre, c'est introduire $\mathrm{du}$ nouveau dans de l'ancien qu'il faut alors réorganiser », écrire serait introduire du nouveau dans du déjà écrit qu'il faut alors réorganiser. Comment se comporte ce qui doit être dit lorsqu'il est confronté à cette nécessaire ré-articulation imposée par le déjà écrit? Mais, plus précisément, comment le scripteur se comporte-il face à son propre déjà écrit?

\section{1. Les opérations d'écriture et le déjà écrit}

Il semble que ce qui est déjà écrit agit sur l'ensemble de la production écrite en y suscitant de profondes modifications. Au fur et à mesure que l'écriture progresse, le volume du déjà écrit s'impose au point de faire ou défaire l'ensemble. Ce volume du déjà écrit amène le scripteur à se positionner : laisse-t-il ce volume sans y revenir, ou au contraire, va-t-il le prendre sans cesse en considération pour y revenir, pour le travailler au corps ? Il s'en suit alors une sorte de dialogue entre ce qui est déjà écrit et ce qui va être écrit. C'est ce déjà écrit qui, pour diverses raisons, formera, à un moment donné, le texte dans sa version finale.

S'appuyant sur l'existence du déjà écrit, il est alors possible de poser un doublement des opérations proposées par l'ITEM (tableau 6) ; nous n'avons plus alors seulement quatre opérations, mais bien huit opérations selon qu'elles apparaissent à la suite du déjà écrit ou bien par retour dans le déjà écrit.

18. Il n'entre pas dans le cadre de ce travail d'introduire plus précisément à la notion de constructivisme. Pour autant, il est guère possible de faire l'économie de rappeler que ce dernier, en tant que théorie générale de l'apprentissage (l'individu reste responsable de ses processus de reconstruction), ne cesse de dialoguer avec le cognitivisme qui considère principalement les processus intra-individuels: les deux ont en partage le concept de processus. 


\begin{tabular}{|c|c|c|c|}
\hline \multicolumn{4}{|c|}{ Les différents types d'opérations d'écriture } \\
\hline & $\begin{array}{l}\text { Opérations d'écriture } \\
\text { à la suite du déjà écrit }\end{array}$ & $\begin{array}{l}\text { Opérations d'éc } \\
\text { par retour dans }\end{array}$ & \\
\hline \multirow{4}{*}{ 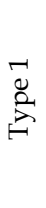 } & Ajout & Ajout & \multirow{4}{*}{ 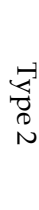 } \\
\hline & Suppression & Suppression & \\
\hline & Remplacement & Remplacement & \\
\hline & Déplacement & Déplacement & \\
\hline
\end{tabular}

Tableau 6.

Les différents types d'opérations d'écriture

Les opérations d'écriture à la suite du déjà écrit sont dites de type 1, alors que celles produites par retour dans le déjà écrit sont dites de type 2 . L'avantage de ce genre de terminlogie est qu'il peut être prolongé sans restriction : il existe bien des opérations de type 3 en ce sens que certaines opérations sont produites à la suite d'un déjà écrit lui-même produit par retour dans du déjà écrit.

Nous avons donc un ajout1, une suppression1, un remplacement1 et un déplacement1, comme nous avons un ajout2, une suppression2, un remplacement2 et un déplacement2 ; mais nous avons aussi un ajout3, une suppression3, un remplacement3 et un déplacement3...

Cette terminologie fait un écho très clair tant à celle de la génétique du texte qu'à celle de la psychologie cognitive. Ainsi l'expression variantes d'écriture recouvre les opérations d'écriture de type 1 , tandis que les opérations d'écriture de type 2 se retrouvent très facilement derrière l'appellation de variantes de lecture telle qu'elles apparaissent chez les chercheurs de l'ITEM.

Du côté de la pyschologie cognitive ${ }^{19}$, il y a aussi des effets de résonnance dans les termes de révisions précontextuelles et révisions contextuelles. Ainsi, Lindgren et Sullivan (2006) parlent de Pre-contextual Revision $^{20}$ pour parler des opérations de type 1 et de Contextual Revisions ${ }^{21}$ pour parler des opérations de type 2 .

19. Pour avoir une vision exhaustive des phénomènes concernés par le processus de révision, voir Alamargot et Chanquoy (2001), notamment le chapitre 3, intitulé Revising process (97-121).

20. «Online revisions can occur at the end of the current text, that is, at the end of the text produced thus far. In the literature these revisions have been called revisions at the point of inscription (Matsuhashi, 1987). These revisions occur as a result of an on-going text production process that is constantly in a state of development » $(2006,160)$

21. «Contextual revisions are defined as revisions undertaken when writers move away from the point of inscription to insert new text or to delete, substitute or rearrange already writen text. » $(2006,171)$ 
Apparaît ici une des caractéristiques de la production écrite enregistré en temps réel. D'un côté, est pris en compte le nombre de retour dans le déjà écrit; lequel permet de se demander combien de retours dans le déjà écrit ont été effectués durant la durée de la production. De l'autre, est pris en compte le nombre d'opérations d'écriture activées: sur les 8 (ou 12, ou 16...) possibles, combien sont effectivement activées? Par défaut, il serait très intéressant de s'interroger sur les opérations d'écriture non activées, l'activité d'écriture restant, par nature, une activité (de production) autoinitée.

\section{2. Conventions de transcription du déjà écrit}

Cette tentative de visualisation du déjà écrit s'inscrit à la suite du vaste ensemble des études consacrées à l'oral ; études pour lesquelles il était évident que pour mieux étudier la production, il fallait non seulement enregistrer mais aussi transcrire.

Pour les études consacrées à la production écrite, il s'agit d'apprendre un réflexe : celui d'enregistrer ce qui est, par nature, fugitif, pour ensuite, tenter de garder des traces par des procédés de visualisation, par des procédés de transcription adaptés.

Sans chercher à prolonger cette ressemblance entre matière écrite et matière orale, toutes deux évidemment ${ }^{22}$ produites en temps réel, il est nécessaire néanmoins de souligner que le métalangage utilisé dans ce genre relativement récent de corpus écrit a du mal à se dégager d'une terminologie forgée pour l'oral et peu idoine pour le matériel de production écrite.

Il est très important d'attirer l'attention sur le fait que les logiciels $\mathrm{d}^{\prime}$ enregistrement $\mathrm{du}$ processus d'écriture offrent diverses manières de rétablir, de restituer cet enregistrement : ils donnent à (re-)voir, certes, le film restitué fidèlement en temps réel de la production écrite, mais pas seulement. Et sans tenir à cet aspect, spectaculaire il est vrai, serait réduire considérablement les possibilités offertes par ce genre de matériel. Ainsi, par exemple, ScriptLog offre, selon les propres mots de son concepteur Sven Strömqvist, "un fichier numérique contenant une représentation exhaustive [le $\mathrm{Log}]$ des événements surgis pendant l'activité d'écriture avec leur distribution temporelle » $(2006,46)$; les données de ces fichiers, notées en chronologie absolue, forment le cœur des logiciels dédiés au temps réel. C'est à partir de ce genre de fichier (voir Annexe) que la restitution filmée est possible, et qu'il est possible de re-construire des transcriptions détaillées.

Il s'agit maintenant de considérer ce que pourrait être des conventions de transcription pour le processus scriptural. Celles qui vont suivre (tableau 7) se concentrent principalement sur la reproduction du déjà écrit à partir des opérations d'écriture et des mouvents du curseur. Elles ne se

22. L'expression de temps réel est réservée aux productions écrites, bien que, de fait, elle désigne toute forme de production, que celle-ci soit orale ou écrite. 
veulent donc nullement exhaustives. L'important est de faire apparaître une proposition de conventions de transcription pour la production verbale écrite .

\begin{tabular}{|c|c|c|c|}
\hline \multicolumn{2}{|c|}{$\begin{array}{c}\text { Conventions } \\
\text { de transcription }\end{array}$} & $\begin{array}{l}\text { Opérations de type } 1 \\
\text { à la suite } \boldsymbol{d} u \text { déjà écrit }\end{array}$ & $\begin{array}{l}\text { Opérations de type } \\
\text { par retour dans le déjà écrti }\end{array}$ \\
\hline$\stackrel{\varpi}{\varpi}$ & Ajouts & $\begin{array}{l}\text { Ajout } 1 \\
\text { Simple }\end{array}$ & $\begin{array}{l}\text { Ajout } 2 \\
(\text { Simple }+) \text { Gras }\end{array}$ \\
\hline 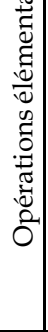 & Suppressions & $\begin{array}{r}\text { Supppressions 1 } \\
\text { Suppression } \\
\text { immédiate - } \\
\text { Barrée avec italique } \\
\text { Suppression différée - } \\
\text { Barrée sans italique }\end{array}$ & $\begin{array}{l}\text { Suppressions } 2 \\
\text { - Suppression immédiate } \\
\text { - Suppression différée } \\
\text { Barree-sans italique-en } \\
\text { gras }\end{array}$ \\
\hline 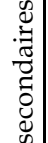 & Remplacements & $\begin{array}{r}\text { Remplacement } 1 \\
\text { Remplacéremplaçant }\end{array}$ & $\begin{array}{l}\text { Remplacement } 2 \\
\text { Remplacéremplaçant }\end{array}$ \\
\hline 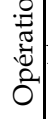 & Déplacements & $\begin{array}{r}\text { Déplacement } 1 \\
\text { Déplacédéplaçant } 1\end{array}$ & $\begin{array}{l}\text { Déplacement } 2 \\
\text { Déplacédéplaçant } 2\end{array}$ \\
\hline
\end{tabular}

Tableau 7.

Conventions de transcription du déjà dit

La sémiologie de ces conventions se veut simple : quatre caractères sont pris en compte: le simple, le barré, l'italique et le gras, en cherchant à associer, autant que possible, un caractère à un geste.

Deux grands principes soutiennent ces conventions. Le premier est la visualisation sans ambiguïté du retour dans le déjà écrit, qui se voit associé un caractère propre, celui du gras. Ainsi, les opérations de type 2 (ou 3...) sont toutes transcrites en caractères gras. Il serait bien évidemment possible de démarquer les opérations de type 2 de celles de type 3 , en associant à ces dernières un caractère particulier : le souligné.

Le second principe est le parti pris systématique d'une chronologie relative au détriment d'une chronologie absolue : les opérations ne sont donc pas transcrites en référence au temps qui s'est écoulé depuis le début de l'écriture mais en référence à l'énonciation en cours, champ pertinent pour le linguiste.

Ces conventions appellent quelques remarques relatives aux gestes. Tout d'abord, au geste d'ajouter est associé le caractère sans variation, le caractère simple de la police. 
Ensuite, au geste multiple de supprimer est associé le caractère barré ; si cette suppression est immédiate (ce qui est, de loin, le cas le plus fréquent), le caractère barré est doublé de celui de l'italique. Mais s'il s'agit d'une suppression différée, seul le caractère de l'italique est pris en compte.

Enfin, les gestes de remplacer et de déplacer, ne font que doubler ceux d'ajouter et de supprimer : ils sont le simple reflet des opérations composées, et ne peuvent donc prétendre à des caractères propres, indépendamment de ceux déjà utilisés.

Voici un texte où le scripteur répond à une tâche qui relève de la spontanéité ${ }^{23}$ en cherchant à prendre le scripteur sur le vif, au sein de son inquiétude première ; la tâche d'écriture est, en cela, limitée à 15 minutes. Pas davantage. Elle cherche à prendre un simple échantillonnage de sa production. Soit le texte suivant, considéré comme un produit final :

\section{Environnement idéal}

Qu'est-ce qui me vient en tête spontanément quand on me donne la tâche de décrire un environnement idéal ? Beaucoup de verdure, peu de pollution, suffisamment d'espace pour que tout un chacun se trouve à l'aise avec soimême et avec les autres. Le climat? Je dois dire que les saisons distinctes et variées typiques du Nord me plaisent, etv que c'est quelque chose que je voudrais avoir dans un environnement idéal. Autrement dit, un été chaud et ensoileillé sans oublier évidemment les journées lumineuses et extrêmement longues comme en Finlande en été, un hiver blanc, avec du soleil et de la neige, et encore deux saisons de "transmission", de passage on peut dire, pour passer du froid au chaud, ou inversement: le printemps et l'automne.

S'il n'est pas possible, pour moi, d'imaginer une vie idéale sans la nature telle que je la connais dans mon pays natale, il m'est tout aussi impossible d'imaginer une existence heureuse sans la présence des enfants et des

23. L'idée est que ce qui se passe en un court instant est représentatif de ce qui se passe sur un plus long temps: pouvoir enregistrer le fonctionnement de l'avant-texte saisi en temps réel dans des productions brèves et spontanées pour mieux pouvoir, par la suite, exploiter son fonctionnement dans des productions plus longues et plus contraintes telles que le résumé de textes ou toutes autres productions de type scolaires (comptes rendus, commentaires, dissertations...) ou universitaires (mémoires, articles scientifiques...). La spontanéité de la production a, selon une définition psychologique, le sens de ce qui n'implique pas le recours de la conscience sur elle-même.

La consigne a été donnée en langue maternelle, soit en finnois : Millainen voisi olla ihanteellinen elinpaikka ja -ympäristö sekä siihen mahdollisesti kuuluvat muut ihmiset/olennot, jos toive olisi toteutettavissa ilman minkäänlaisia rajoituksia? Voit kirjoittaa tekstin valintasi mukaan joko yksikön ensimmäisessä (minä), yksikön toisessa (sinä) tai yksikön kolmannessa (hän) persoonassa.

Elle peut être traduite de la manière suivante: Décrivez votre environnement idéal ainsi que les personnes ou les êtres qui pourraient y vivre. Imaginez-le sans vous limiter. Vous pouvez écrire à la première personne (Je), ou à la deuxième (Tu), ou bien encore à la troisième (Il/Elle). 
animaux. Les enfants et les animaux font partie de la nature.

Voici, à titre de simple illustration, ce que pourrait être une transcription $\mathrm{du}$ processus scriptural du déjà dit du texte précédent, à partir des opérations d'écriture et des mouvements du curseur :

\section{Environñement idéal}

Que me vionet'est-ce qui me vient en tête spontanément quand on me donne la tâche d'e décrire un environnement idéal $?^{18}$ Be ${ }^{1} a^{2}$ coup de verdure $\leftarrow^{1} \rightarrow^{2}$, peu de pollution, suffisamment $d^{\prime}$ espace pour que thotout un chacun se trouve

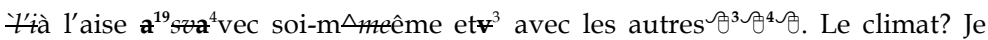
dois dire que les saisons disctinctes et variées typiques du Nord mae plaisent ${ }^{5}{ }^{5} \exists$, etv que $\overline{c e}^{\prime}$ est vquelque chose que nje je voudrais avoir dans un $\mathbf{u e}^{10}$ nvironnement idéal. DoAutrement $\mathbf{n}^{11}$ dit, un été chaud et ensoileillé aveesans oublier évidemment les journées lumineuses et ex trêmement longues ${ }^{6} \mathbf{0}$, †acomme en Finlande en été, un hiver blanc, avec du $\mathbf{d s} \mathbf{s}^{12}$ oleil et de la naigeeidge, et encore dueux ${ }^{13}$ saisioons de "transmisson", de passage on peut dire, pour passer du froid, ${ }^{14}$ au chaud ou läinverseinversemen $x t t$ : le prinpntemps et l'aiutomne.

He ne peuxS'il n'est pas possible, pour moi, d'imaginer une vie idéale sans la nature telle que je la connais dans mon pays natale, il $\mathbf{n m}^{7}{ }^{7}$ '̈e'est égatout aussi $\mathrm{im}^{8}$ possible non plus pou $\mathrm{e}^{9} \hat{0}^{7 \beta^{8} \beta^{9} *} \mathrm{~d}^{\prime}$ imaginetrr un-eviee existence $^{15}$ heureuse sans la p: ${ }^{16}$ résence des enfants et des animaux. $\hat{\beta}^{10} \beta^{11} \beta^{12} \beta^{13} \beta^{14} \beta^{15} \beta^{16} \beta^{17}$ ? $f$. Les enfants et les animaux font partie de la nature an sens. $18 \sqrt[3]{19} \sqrt{3}$

Toute-nie, jä'a/'J'ai toujours vécu au bord de la mer:/m, ou plus précisément, je vis mes hivers au bord de 1 amer proprement dite et mes étés à lane campagne au bord d'un grand lac. Cette présence de l'eau dans mon environnement $\bar{q}$ est quelque chose qui me manque lorsque je suis

\section{Que dit $^{24}$ cette transcription?}

En observant cette transcription, il est aisé de répondre à des questions de type :

1) Combien de manipulations des périphériques ont été faites ?

Les manupulations de la souris sont au nombre de dix-neuf; celles des flèches sont au nombre de deux. Aucune manipulation du pavé tacile est notée.

2) Y-a-t-il eu des retours dans le déjà écrit ? Et si oui, combien ?

Oui, la transcription fait apparaitre de nombreux retours dans le déjà écrit, presque tous effectués à partir du périphérique de la souris. Le scripteur est revenu dans son déjà écrit par 19 fois.

3) De quels types sont les opérations d'écriture activées ?

24. Ce que cette transcription ne dit pas : cette transcription a le défaut de toutes les transcriptions. A force de vouloir dire beaucoup, elle finit par en dire trop (peu). Elle se doit de choisir entre précision et lisibilité. Elle est restreinte à faire des choix. Et ce qui a été fait. 
Des opérations de type 1 (ajouts, suppression et remplacements). Mais aussi des opérations de type 2 (ajouts, suppressions et remplacements). Il serait même possible de déceler une suppression de type 3 (ligne 1: Que me vionet'), puisque le scripteur procède à une suppression à la suite d'un déjà écrit (inscription de Qu-) luimême produit par retour dans du déjà écrit.

4) L'ordre de lecture du texte produit correspond-il à l'ordre d'écriture ?

Non. La première phrase du texte fini a été rédigée en fin d'écriture ; ce qui est intéressant, puisque la première phrase mentionne explicitement l'adverbe spontanément. Apparaît ici toute la stratégie du scripteur, toute sa ruse à prétendre du spontané là où il y a du calculé, de l'artificiel. Cette écriture se situe, pour reprendre les mots d'Irène Fenoglio, « du côté de la maîtrise », elle est « jouée » $(2001,178)$.

Il est facile de voir ce que la didactique de la production écrite pourrait tirer de ce genre de transcription qui met en scène visuellement le rôle du déjà écrit en sein du processus d'écriture. Il est tout aussi aisé de comprendre ce qu'une telle didactique pourrait tirer de la méthodologie proposée par la génétique .

\section{De la génétique à la didactique}

Il est non seulement possible, mais aussi souhaitable, d'exploiter ce type d'approche dans des séquences qui se veulent des lieux d'apprentissage de l'écriture. A force de prétendre, peut-être d'une manière trop péremptoire, que le texte est le lieu où se joue l'écrit, il devient parfois trop facile d'oublier que l'écriture pourrait être un objet d'enseignement à part entière.

\subsection{Orientations générales}

Il semble tout naturel à l'enseignant de commencer par lui-même. Dans une démarche résolument réflexive, celui-ci a tout intérêt de se considérer lui-même en train d'écrire, de se prendre par la main pour voir agir sa ou ses propres mains. Par l'intermédiaire d'un logiciel d'enregistrement de l'écriture en temps réel, il peut essayer de visualiser ce qui se passe lors de son écriture. Comment cette écriture qui lui est propre, procède-t-elle ? Les retours dans le déjà écrit sont-ils récurrents comme il est possible de s'y attendre lors de productions expertes?

Après quelques heures de manipulation du logiciel, il est alors tout à fait possible de se risquer à faire des transcriptions et de voir comment sa propre écriture procède. La pratique donc des transcriptions de l'écriture commence par la pratique de sa propre écriture, afin de se sensibiliser à la démarche.

Cela acquis, l'enseignant en écriture a tout loisir de proposer des activités diverses pour exploiter la richesse didactique de la production en temps réel. 
Kirk P. H. Sullivan et Eva Lindgren (2006) en propose quelques unes allant de discussions ${ }^{25} \mathrm{~s}^{\prime}$ appuyant sur les enregistrements des productions des étudiants à la mise à disposition ${ }^{26}$ des étudiants de leurs propres enregistrements via un logiciel de temps réel : les étudiants peuvent ainsi tirer d'énormes profits à voir ainsi se dire leurs propres textes.

Une dernière remarque qui tire profit de la grande diffusion d'un document comme le Cadre Européen Commun de Référence parmi les enseignants de langues étrangères. Dans cet ouvrage consacré à apprendre à évaluer des textes produits, il est néanmoins réservé quelques pages aux stratégies de production $(2001,48)$. Comment alors ne pas faire le lien entre nos deux propos et de voir que les études des productions et celles des produits ont tout intérêt à finir par se rencontrer.

Est-il encore possible de continuer de parler de(s) stratégies de (la) production écrite en faisant l'économie des approches liées au temps réel ? Car il est possible d'apprendre, d'enseigner, et d'évaluer à partir des opérations d'écriture.

\section{2. Catégorisations opérationnelles}

Après avoir une vision complète, sinon exhaustive, des opérations engagées, par chaque scripteur, via une transcription, il convient de faire une description clinique de toutes les opérations effectuées par un scripteur durant le temps qu'a duré l'enregistrement ; ce qui est loin d'être aisé.

Comment alors catégoriser ce qui se passe au sein des successions d'opérations ? Comment pouvoir mettre en évidence ce qui relève d'un simple respect de la norme et ce qui relève de libres choix, d'un vouloirécrire qui se cherche? Pour essayer d'apporter une amorce de réponse, il serait tentant de schématiser dans un tableau (8) quelques grandes lignes :

\begin{tabular}{|c|l|l|l|}
\hline \multicolumn{3}{|c|}{ Catégorisation opérationnelle (chronologie absolue) } \\
\hline \multicolumn{2}{|c|}{ Opérations d'écriture de type 1 } & Opérations d'écriture de type 2 (et 3...) \\
\hline Respect de la norme & Vouloir-écrire & Respect de la norme & Vouloir-dire \\
\hline
\end{tabular}

Tableau 8.

Catégorisation opérationnelle

25. «An analysis of a learner's keystroke log could [...] provide the teacher with the information about which features a learner has noticed and could be discussed as their level of salience would already be High » $(2006,205)$.

26. «[...] make the keystroke log replay function directly available to the learner to examine and reflect upon individually in an in-class or out-of-class setting. The learner can write diary entries, write course work or, in fact, any document that needs to be writen using keystroke logging software. The student can once they have completed their document, replay the log, and among other things, reflect upon the process and notice features of the writing process, grammatical feature about which they are insecure. » $(2006,205)$. 
Chaque opération formelle est, à son tour, prise pour elle-même et donne lieu à des catégorisations internes: s'agit-il d'une opération formelle motivée par le soucis d'adaptation à la norme, tant linguistique (orthographique ou morphosyntaxique), que pragmatique (textuelle, énonciatif...), ou bien s'agit-il d'une opération formelle motivée par l'expression d'un vouloir-écrire (initial) qui cherche à se construire autant dans ses continuités que dans ses ruptures? Dans les deux cas, se manifestent des hésitations, des reformulations.

Cette schématisation ne cherche pas profondément à s'éloigner des deux grands modèles de la révision en production verbale écrite: ni de celui de Faigley et Witte (1981) qui fut durant longtemps la référence en matière, où les termes de Surface changes et Text-base changes se retrouvent dans ceux de Respect de la norme et Vouloir-dire ; ni de celui de Lindgren et Sullivan (2006), modèle récent de la révision en temps réel, où les termes de Form revision et Conceptual revision se retrouvent directement dans ceux de Respect de la norme et Vouloir-dire.

Afin d'illustrer ce que permet une catégorisation détaillée d'opération, va suivre une analyse de l'opération de remplacement effectuée à la suite du déjà écrit.

\subsection{L'opération de remplacement : une opération didactique}

Le rôle joué par l'opération de remplacement effectuée à la suite du déjà écrit est loin d'être mineur. Il semblerait que cette opération soit celle sur laquelle se focalisent bon nombre d'enjeux génétiques sur lesquels une didactique de l'écriture pourrait se construire.

Ces remplacements de lettres (et non pas de segments) de type 1 sont classés en deux groupes selon qu'ils sont effectués après l'écriture complète d'un mot (4 possibilités), ou bien pendant l'écriture d'un mot (deux possibilités).

Dans le premier cas de figure, le remplacement intervient à la fin de l'écriture d'un mot.

- Première possibilité : une lettre (ou des lettres) manque(nt).

Description du geste effectué : suppression de la (des) lettre(s) jusqu'à l'endroit en question et ajout contigu de la (des) lettre(s).

Geste alternatif en un temps: Curseur gauche et ajout d'une (des) lettre(s).

$$
\text { Exemple }^{27} 1 \text { : peitetite (où il manque un } t \text { entre } e \text { et } i \text { ). }
$$

- Deuxième possibilité : une lettre (ou des lettres) est (sont) en trop. Description du geste effectué : suppression de la (des) lettre(s) jusqu'à l'endroit en question et ajout contigu de la (des) lettre(s).

27. Les exemples qui suivent sont tous issus d'un corpus de production écrite d'étudiants finnophones de première année universitaire (français langue étrangère). 
Geste alternatif en un temps: curseur gauche/suppression de la (des) lettre(s) en trop.

Exemple 2: vou drais (où le $i$ est en trop).

- Troisième possibilité : aucune lettre en trop ou en moins.

Description du geste effectué : suppression d'une (des) lettre(s) et ajout contigu de la (des) même(s) lettre(s).

- Il n'existe probablement pas de geste alternatif.

Exemple 3 : La a (où $L a$ redevient $L a$ ).

- Quatrième possibilité : inversion complète de (deux) lettres.

Description du geste effectué : suppression de la (des) lettre(s) jusqu'à l'endroit en question et ajout contigu de la (des) lettre(s) dans le bon ordre (+ ajout d'autres lettres).

Geste alternatif en deux temps: curseur gauche/suppression de la première lettre + curseur droit/ajout contigu de la même lettre.

Exemple 4a : dasn ns (où l'ordre sn devient $n s$ ).

- Variante du précédent: inversion dans le redoublement des lettres.

Description du geste effectué : suppression de la (des) lettre(s) et ajout contigu de la (des) lettre(s) sans redoublement.

Geste alternatif en deux temps: curseur gauche/ajout d'une lettre + curseur droit/suppression d'une (des) lettre(s).

Exemple $4 \mathrm{~b}$ : ausïisi (où ii devient ss).

Le second cas de figure est représenté par un remplacement intervenant au milieu de l'écriture d'un mot. Dans les deux possibilités, le clavier de type qwerty reste problématique pour l'apprentissage d'une langue comme le français ou tantôt la proximité de lettres peuvent gêner (la lettre $r$ et la lettre $e$ se touchent), ou tantôt l'accentuation demande des manipulations supplémentaires.

- Première possibilité : une lettre $\mathrm{x}$ est remplacée par une autre lettre $\mathrm{y}$ avec suppression (totale ?) de $x$.

Il n'existe probablement pas de geste alternatif.

Exemple $5: \mathrm{J} r e$ (où $r$ disparait au profit de $e$ ).

- Deuxième possibilité : des lettres, ou des accents, dans un réel désordre.

Description du geste effectué : suppression de la (des) lettre(s)/accent(s) jusqu'à l'endroit en question et ajout contigu de la (des) lettres/accent(s).

Il n'existe probablement pas de geste alternatif.

Exemple 6: t'rès (où l'accent grave vient trop rapidement).

La mention des gestes alternatifs permet de mettre en évidence certaines stratégies de manipulation du clavier : ils sont gradués sur une échelle de quatre niveaux : aucun, en un temps, en deux temps, en trois temps. Autant il est aisé de comprendre l'économie de geste réalisée pour les deux dernières graduations (faire moins de gestes), autant pour la 
seconde (un geste équivalent à un autre geste), le choix effectué par le scripteur est intéressant. Il existe même des cas où il n'existe aucune autre possibilité de s'y prendre pour refaire ce remplacement. Par exemple, le cas de réécriture du même par le même... ces cas semblent riches pour essayer de comprendre la manifestation d'un vouloir-dire.

Il s'agit bien de faire apparaître des données périssables, mais fondamentales pour qui veut comprendre, aider à comprendre ce qui se passe en aval de l'écriture, avant que celle-ci se transforme en de l'écrit; et l'opération de remplacement offre un cadre simple à qui veut prendre le temps de regarder. Indépendamment des cas de désordres (exemple 6) issus de manipulations mal aisées du clavier et des cas de suppressions réelles (exemple 5), les autres cas de remplacement permettent de souligner la richesse du fonctionnement de ces remplacements (exemples $4,3,2$ et 1) qui se produisent alors que l'écriture du mot n'est pas encore achevée.

A chaque fois une part ajoutée exige pour apparaître sa part supprimée laquelle est une forme d'anticipation sur la première. Il s'agit de faire à soi-même une première tentative, provisoire, pour faire exister une forme plus permanente, plus proche de ce qui veut être dit. Cette part ajoutée, immédiatement successive, ressemble à sa part supprimée. Il faut tenter, oser une première forme pour pouvoir la reprendre immédiatement sur le mode de l'analogie afin d'obtenir une autre forme plus satisfaisante, plus permanente. Un signifiant s'installera momentanément et rapidement un autre viendra prendre sa place. C'est précisément dans ce jeu de signifiants successifs que réside toute la dimension de l'écriture.

\section{Conclusion}

Il est maintenant possible d'apprendre à considérer un corpus de production verbale écrite à partir d'un nombre de minutes (et d'heures) en cours de production, et pas seulement à partir d'un nombre de pages données.

Les opérations d'écriture, associées aux mouvements du curseur activés par l'intermédiaire d'une souris ou équivalents (flèches et pavé tactile), sont des invariants processuels, ou opérationnels, auxquels aucun scripteur n'échappe lors de son écriture. Les productions verbales qui en sont issues se situent en deçà de pratiques prétendument bonnes ou mauvaises, en deçà de pratiques expertes, ou novices, et en deçà de pratiques pathologiques ou déficientes, que celles-ci soient écrites en langues maternelle, étrangère ou seconde.

Chaque scripteur se positionne, tout d'abord, dans sa singularité qui lui est propre, face à son déjà écrit, c'est-à-dire face au volume de son écriture en mouvement, construite à partir de ses ajouts, ses suppressions, ses remplacements et ses déplacements. Accepter de se mettre à écrire, c'est accepter cette confrontation avec soi-même, avec ses continuités et 
ses ruptures. Chaque individu y fait face à son propre lot d'hésitations, de reformulations et de recherches. Il s'avère ainsi qu'une écriture plus expérimentée reviendra plus souvent encore dans son déjà écrit pour, principalement, y effectuer des ajouts et des remplacements.

Que peut-on souhaiter? Que, dans un avenir récent, soit mise en place une numérisation de corpus nationaux, une fois admise l'utilisation d'ordinateurs pour écrire, lors d'examen ou de concours relevant d'une autorité nationale, à l'image de l'utilisation maintenant banalisée des calculatrices pour certaines épreuves ; et que, dans un avenir plus lointain, soit mise sur place une diffusion des technologies du temps réel.

Mais, comme le souligne Sylvie Plane $(2004,164)$, il est question de se rappeler que l'enseignement de l'activité de la production écrite reste une activité récente. La présence de la critique génétique, au sein de ces activités d'enseignement, ne peut se réduire à l'étude du fonctionnement des écritures des autres, elle autorise aussi des transpositions permettant une pratique de l'écriture de soi.

Annexe : Extrait d'un fichier numérique de ScriptLog

Les 30 premières secondes d'écriture correspondant à la transcription.

\begin{tabular}{|c|c|c|c|c|}
\hline time & type & from & to & key \\
\hline 0.00 & 10 & 1 & 0 & $<$ START $>$ \\
\hline 5.75 & 7 & 0 & 0 & \\
\hline 6.68 & 7 & 1 & 1 & $\mathrm{n}$ \\
\hline 7.20 & 7 & 2 & 2 & $\mathrm{v}$ \\
\hline 7.91 & 7 & 3 & 3 & $\mathrm{i}$ \\
\hline 8.08 & 7 & 4 & 4 & $\mathrm{r}$ \\
\hline 8.20 & 7 & 5 & 5 & o \\
\hline 8.45 & 7 & 6 & 6 & $\mathrm{n}$ \\
\hline 8.60 & 7 & 7 & 7 & $\mathrm{n}$ \\
\hline 8.73 & 7 & 8 & 8 & $\mathrm{a}$ \\
\hline 10.06 & 5 & 9 & 9 & $<$ DELETE $>$ \\
\hline 10.63 & 7 & 8 & 8 & $\mathrm{e}$ \\
\hline 10.80 & 7 & 9 & 9 & $\mathrm{~m}$ \\
\hline 10.90 & 7 & 10 & 10 & e \\
\hline 11.01 & 7 & 11 & 11 & $\mathrm{n}$ \\
\hline 11.21 & 7 & 12 & 12 & $t$ \\
\hline 11.45 & 7 & 13 & 13 & \\
\hline 11.68 & 7 & 14 & 14 & $\mathrm{i}$ \\
\hline 11.95 & 7 & 15 & 15 & d \\
\hline 12.31 & 7 & 16 & 16 & é \\
\hline 12.71 & 7 & 17 & 17 & a \\
\hline 12.78 & 7 & 18 & 18 & 1 \\
\hline 21.46 & 7 & 19 & 19 & $<\mathrm{CR}>$ \\
\hline 22.75 & 7 & 20 & 20 & $<\mathrm{CR}>$ \\
\hline 26.28 & 7 & 21 & 21 & B \\
\hline 26.71 & 7 & 22 & 22 & $\mathrm{e}$ \\
\hline 26.88 & 7 & 23 & 23 & $\mathrm{u}$ \\
\hline 27.00 & 7 & 24 & 24 & a \\
\hline 27.33 & 7 & 25 & 25 & $\mathrm{c}$ \\
\hline 27.75 & 7 & 26 & 26 & o \\
\hline 27.88 & 7 & 27 & 27 & $\mathrm{u}$ \\
\hline 28.10 & 7 & 28 & 28 & $\mathrm{p}$ \\
\hline 28.26 & 7 & 29 & 29 & \\
\hline
\end{tabular}




\begin{tabular}{|lllll|}
\hline 28.46 & 7 & 30 & 30 & $\mathrm{~d}$ \\
28.65 & 7 & 31 & 31 & $\mathrm{e}$ \\
28.75 & 7 & 32 & 32 & $\mathrm{v}$ \\
28.91 & 7 & 33 & 33 & $\mathrm{e}$ \\
29.03 & 7 & 34 & 34 & $\mathrm{r}$ \\
29.28 & 7 & 35 & 35 & $\mathrm{~d}$ \\
29.56 & 7 & 36 & 36 & $\mathrm{u}$ \\
29.68 & 7 & 37 & 37 & $\mathrm{r}$ \\
29.98 & 7 & 38 & 38 & \\
\hline
\end{tabular}

Time (colonne de gauche) note la chronologie absolue. Type (deuxième colonne à partir de la gauche) repésente le type d'événement: par exemple, le chiffre 7 représente l'écriture de minuscules. Les mentions de from à to représentent la position sur l'écran. La succession des opérations d'écriture est à retrouver à partir de la colonne de droite qui doit se lire verticalement. La chronologie relative n'est pas notée explicitment par le logiciel, elle doit être reconstruite.

La mention de $<$ DELETE $>$ note la suppression d'une lettre, tandis que celle de $<\mathrm{CR}>$ note un alinea.

\section{Bibliographie}

Alamargot, D. et Chanquoy, L. (2001), Through the Models of Writing. Studies in Writing. Dordrecht, Boston, London : Kluwer Academic Publishers.

Boré, C. (2000) « Le brouillon, introuvable objet d'étude ? », Pratiques 105-106, $23-49$.

Cadre Européen Commun de Référence: Apprendre, Enseigner, Evaluer. (2001), Conseil de l'Europe, Division des politiques linguistiques. Paris : Didier.

Chanquoy, L. et Fenoglio, I. (2007), « la notion d'avant-texte : point de rencontre pour une compréhension de l'écriture en acte ", Langue Française 155, p. 3-7.

Fabre, C. (1987), Les Activités métalinguistiques dans les écrits scolaires, Thèse de doctorat d'Etat ès Lettres, Universités Descartes Paris V.

Faigley, L. et Witte, S. (1981), «Analyzing revision», College Composition and Communication 32, 400-414.

Fenoglio, I. (2001) «Les événements d'énonciation: Focaliseurs d'interprétation pyschanalytique, matériau pertinent de l'analyse linguistique d'énonciation », M. Arrivé et C. Normand (éds) Linguistique et psychanalyse, Colloque international de Cerisy-La-Salle, Septembre 1998, 167-184.

Doquet-Lacoste, C. (2004), Etude génétique de l'écriture d'élèves de Cours Moyen 2, année 1995-96, Thèse de Doctorat de Sciences du Langage soutenue à l'Université de Paris 3 le 17 juin 2003, Lille : Atelier National de Reproduction des Thèses.

(2006) «L'objet insaisissable: l'écriture sur traitement de texte", Genesis 27, $35-44$.

Grésillon, A. (1994), Eléments de critique génétique. Paris: Presses Universitaires de France.

Grésillon, A. et Lebrave, J.-L. (2008), « Linguistique et génétique des textes : un décalogue ", Le français moderne, Tendances actuelles de la linguistique française, Paris, ?, 37-49.

Langue française 155 (2007), Chanquoy, L. et Fenoglio, I (eds) Avant le texte : les traces de l'élaboration textuelle.

Langages 147 (2002), Processes d'écriture et marques linguistiques.

Leblay, C. (2007a), "L'avant-texte comme texte sur le vif. Analyse génétique d'opérations d'écriture en temps réel », Langue française $\mathrm{n}^{\circ} 155$, Paris, 
(2007b), « L'avant-texte: le texte en émoi », in J. Härmä \& U. Tuomarla et alii (éds) Actes du XXIXème Colloque International de Linguistique Fonctionnelle , Publication du Département des Langues Romanes de l'Université de Helsinki 18, Helsinki: 141-146.

Lindgren, E. et Sullivan, K.P.H. (2006), «Analysing Online Revision», in K. P. H. Sullivan et E. Lindgren (éds) Computer Keystroke Logging and Writing, Amsterdam, Elsevier, 147-188.

Matsuhashi, A. (1987), « Revising the plan and altering the text », in A. Matsuhashi (éd.) Writing in real time - modelling production processes, Norwood, NJ : Ablex Publishing company, 197-223.

Plane, S. (2004), «Quelques éclaircissements informatisées et l'apprentissage de l'écriture", in A. Piolat (Ed.) Ecriture. Approches et sciences cognitives, Publication de l'université de Provence, 161-179.

Strömqvist, S., (2006), «Une approche expérimentale du processus d'écriture: l'enregistrement de la frappe au clavier », Genesis 27, 45-58.

Sullivan, K. P. H. et Lindgren, E. (2006), « Supporting Learning, Exploring Theory and Looking Forward With Keystroke Logging ", in K. P. H. Sullivan et E. Lindgren (éds) Computer Keystroke Logging and Writing, Amsterdam, Elsevier, 203-211.

Velay, J.-L., Longcamp, M. \& Zerbato-Poudou, M.-T. (2004), « Le stylo et le clavier. Notre mode d'écriture influence-t-il notre perception de l'écrit ? », in A. Piolat (Ed.) Ecriture. Approches et sciences cognitives, Publication de l'université de Provence, 37-54.

Université de Jyväskylä, Finlande Institut des Textes et manuscrits Modernes (ITEM), Paris 